\title{
Hypolipidaemic and anti-inflammatory effects of fixed dose combination of atorvastatin plus ezetimibe in Indian patients with dyslipidaemia
}

\author{
Biswa Mohan Padhy'1, MD, DM, Rakesh Yadav², MD, DM, Yogendra Kumar Gupta ${ }^{3}$, MD
}

INTRODUCTION We aimed to assess the efficacy of fixed dose combination of atorvastatin plus ezetimibe in Indian patients with dyslipidaemia.

METHODS A double-blind study was conducted to assess the effect of fixed dose combination of ezetimibe $10 \mathrm{mg}$ plus atorvastatin $10 \mathrm{mg}$ on lipid profile, oxidised low-density lipoprotein (ox-LDL), high-sensitivity C-reactive protein (hsCRP) and soluble intercellular cell adhesion molecule (sICAM) in dyslipidaemic patients with or at high risk of coronary artery disease, and compare it with atorvastatin $10 \mathrm{mg}$ monotherapy. 30 patients were randomised to receive ezetimibe plus atorvastatin or atorvastatin once daily for four weeks.

RESULTS Of the 30 patients, 10 men and 5 women (mean age $54.3 \pm 1.6$ years) received ezetimibe plus atorvastatin, while 13 men and 2 women (mean age $53.7 \pm 2.8$ years) received only atorvastatin. The combination treatment significantly reduced total cholesterol (percentage treatment difference $-14.4 \pm 6.5,95 \%$ confidence interval [Cl] -1.0 to $-27.7 ; p=0.041$ ) and LDL cholesterol (LDL-C; percentage treatment difference $-19.9 \pm 6.1,95 \% \mathrm{Cl}-7.4$ to -32.4; $p=0.003$ ) compared to atorvastatin monotherapy. 13 patients on combination treament achieved the National Cholesterol Education Program target for LDL-C as compared to 9 patients on atorvastatin monotherapy $(p=0.032)$. Significant reductions in very low-density lipoprotein cholesterol, triglyceride, ox-LDL and sICAM were observed with combination treatment compared to atorvastatin monotherapy. However, no significant change was seen in high-density lipoprotein cholesterol or hsCRP levels between the two groups.

CONCLUSION Combination treatment with atorvastatin and ezetimibe had relatively better lipid-lowering and antiinflammatory efficacy than atorvastatin monotherapy.

Keywords: atorvastatin, ezetimibe, high sensitivity C-reactive protein, intercellular cell adhesion molecule

Singapore Med J 2013; 54(2): 90-95

\section{INTRODUCTION}

Coronary artery disease (CAD), which afflicts millions of people worldwide, has reached alarming proportions in India. It is estimated that about $52 \%$ of CAD deaths in the country occur among people below 70 years of age, whereas in Western countries, CAD is considered a disease of the aged. ${ }^{(1)}$ The huge burden of CAD in India is due to the high prevalence of CAD risk factors in the population. Dyslipidaemia is a major modifiable risk factor for the development of CAD. ${ }^{(2)}$ High total cholesterol (TC), low-density lipoprotein cholesterol (LDL-C) and triglyceride (TG) levels, along with a low high-density lipoprotein cholesterol (HDLC) level, typify the mixed dyslipidaemia prevalent in the Indian population. ${ }^{(3,4)}$

Several large clinical trials have reported that statins are efficacious in lowering LDL-C, as well as in preventing and reducing mortality due to cardiovascular events in patients with CAD. ${ }^{(5,6)}$ The beneficial effects of statins extend beyond their cholesterol-lowering properties. Its anti-inflammatory, antioxidant, antiproliferative and antiplatelet effects have been suggested to be responsible for the overall efficacy of the drug. ${ }^{(7-9)}$ However, for every subsequent doubling of the statin dose, there is only a mean $6 \%$ incremental reduction in LDL-C, and this is also associated with a higher frequency of adverse effects. ${ }^{(10)}$ The National Cholesterol Education Program-Adult Treatment Panel III (NCEP-ATP III) has recommended a minimum LDL-C reduction of $30 \%-40 \%$ for patients considered to be at moderate to very high risk for CAD, a goal that is not always achievable with monotherapy. ${ }^{(11)}$ Reportedly, less than $60 \%$ of patients with CAD or CAD risk equivalents achieve NCEP goals for LDL-C with monotherapy. ${ }^{(12)}$ To overcome the limited efficacy of single agents and reduce the risk of adverse effects, which are often dose-related, the concept of combination drug therapy with lipid-modifying agents having mechanisms of action that differ from but complement those of low dose statins has emerged as a potential strategy for the management of dyslipidaemia.

Ezetimibe, a selective inhibitor of cholesterol absorption from the small intestine, inhibits cholesterol absorption from the intestine by blocking the action of the cholesterol transporter, Niemann-Pick C1-Like 1 (NPC1L1) protein. ${ }^{(13)}$ Its novel mechanism of action is complementary to that of statins, making it an attractive candidate for combination treatments with statins. Clinical studies have reported that the LDL-C-lowering efficacy of combination

${ }^{1}$ Department of Pharmacology, Vardhman Mahavir Medical College and Safdarjung Hospital, ${ }^{2}$ Department of Cardiology, ${ }^{3}$ Department of Pharmacology, All India Institute of Medical Sciences, New Delhi, India

Correspondence: Dr Biswa Mohan Padhy, Assistant Professor, Department of Pharmacology, Vardhman Mahavir Medical College and Safdarjung Hospital, New Delhi 110029, India.drbisu7@gmail.com 
treatment is superior to statin monotherapy. ${ }^{(10)}$ However, additional reduction in LDL-C is variable and ranges between $6 \%$ and $20 \%$ across different studies. ${ }^{(14)}$ While a majority of the studies on ezetimibe and statin combination therapy have focused primarily on its lipid-lowering efficacy, the superiority of combination treatment over statin monotherapy in terms of its effect on proinflammatory markers is still not well-established. Some studies have reported that the combination of ezetimibe and statin is superior to treatment with statin alone for the reduction of high-sensitivity C-reactive protein (hsCRP). ${ }^{(13-15)}$ However, a few others have noted that hsCRP, proinflammatory cytokines and adhesion molecules remain unchanged with the combination of ezetimibe and statin. ${ }^{(16,17)}$ To the authors' knowledge, only one previous open-label, non-comparative study has reported the lipid-lowering efficacy of ezetimibe monotherapy in Indian patients with familial hypercholesterolaemia. ${ }^{(18)}$ In view of this, a pilot study was undertaken to evaluate the effect of a fixed dose combination (FDC) of atorvastatin and ezetimibe on lipid profile, oxidised LDL (ox-LDL), hsCRP and soluble intercellular cell adhesion molecule (sICAM) in dyslipidaemic patients presenting to a tertiary healthcare centre in urban India, and compare these findings with that of patients receiving atorvastatin monotherapy.

\section{METHODS}

This study was a randomised, double-blind, parallel group, active comparator-controlled clinical trial conducted between June 2009 and March 2010 at the departments of cardiology and pharmacology of the All India Institute of Medical Sciences (AIIMS), New Delhi, India. The sample size for the pilot study was based on previous studies carried out by other authors in different patient populations and clinical settings. ${ }^{(17,19)}$ As LDL-C lowering was consistently reported in earlier studies, it was also chosen as a parameter for our calculations. A sample size of 30 patients was required to detect a difference of $12 \%$ or more in LDL-C between the two patient groups, with a standard deviation of 10 . The power of the study was $80 \%$ and the level of significance was fixed at $p$-value $<0.05$. The study, which was approved by the institutional ethics committee at AIIMS, was carried out in accordance with the principles of the Declaration of Helsinki, Good Clinical Practice and the bioethics guidelines of the Indian Council of Medical Research. Written informed consent was obtained from each patient prior to enrolment. This study was registered with the Clinical Trials Registry-India (CTRI/2010/091/000003).

The participants consisted of men and women aged 18-70 years. The inclusion criteria were: (a) patients with fasting $L D L-C \geq$ $100 \mathrm{mg} / \mathrm{dL}$ and CAD or CAD risk equivalents; or (b) patients with fasting LDL-C $\geq 130 \mathrm{mg} / \mathrm{dL}$ with two or more major risk factors for the development of $\mathrm{CAD}$, having a ten-year absolute CAD risk of $10 \%-20 \%$, on therapeutic lifestyle changes for three months, and who were either drug naive or on low-dose atorvastatin (10 mg once daily). Patients with medical histories of systemic infection within six weeks of study initiation, unstable angina, myocardial infarction, heart failure or stroke within three months of the study, history suggestive of substance or illicit drug abuse, fasting serum TG level $\geq 350 \mathrm{mg} / \mathrm{dL}$, uncontrolled hypertension, uncontrolled diabetes mellitus, severehepatic or renal impairment, malabsorption syndrome or inflammatory bowel disease, on drugs that increase the risk of statin-induced myopathy or on anti-inflammatory or antioxidant therapy within six weeks of study initiation, or those who were pregnant or lactating, were excluded from the study. The withdrawal criteria from the study included the development of serious adverse events or any other medical condition that, according to the investigator, could limit the patient's evaluation or participation, and voluntary withdrawal of consent.

Patients were randomly assigned to either the study group (atorvastatin plus ezetimibe FDC) or control group (atorvastatin only) by means of computer-generated random numbers using a variable block randomisation scheme in the Random Allocation Software version 1 (RAS 2008; Isfahan University of Medical Sciences, Isfahan, Iran). Blinding was maintained by packing the medication in identical-looking, opaque, coded receptacles containing identical tablets of ezetimibe $10 \mathrm{mg}$ plus atorvastatin $10 \mathrm{mg}$, or atorvastatin $10 \mathrm{mg}$.

All the patients underwent thorough physical and laboratory examinations (electrocardiography [ECG], lipid profile, aspartate transaminase $[\mathrm{AST}]$, alanine transaminase $[\mathrm{ALT}]$, serum bilirubin, urea and creatinine) at screening (Day 3). The patients were advised to continue with their existing medications at screening and during the study. On Day 0, patients who satisfied the eligibility criteria were randomly assigned to either the control or study group. For baseline assessment of hsCRP, sICAM and ox-LDL, $2 \mathrm{~mL}$ of blood was collected from the antecubital vein. Patients in the study group received FDC of atorvastatin $10 \mathrm{mg}$ and ezetimibe $10 \mathrm{mg}$ once daily, while patients in the control group received atorvastatin $10 \mathrm{mg}$ once daily for four weeks. The medicines used in the study were provided by Torrent Research Centre, Ahmedabad, India.

All the patients received advice on dietary and lifestyle modifications. The enrolled patients were instructed to take the medications regularly for the entire duration of the study, as well as to maintain the same dietary and exercise schedules during the study. The patients were followed up after four weeks, and blood samples were taken for the estimation of fasting lipid profile, renal function test, liver function test, serum hsCRP, sICAM and ox-LDL. Fasting TC, LDL-C, HDL-C, very low-density lipoprotein cholesterol (VLDL-C), TG, AST, ALT, blood urea and serum creatinine were estimated by standard enzymatic colourimetric method using an autoanalyser. Commercially available enzymelinked immunosorbent assay (ELISA) kits were used for the estimation of hsCRP (BioCheck Inc, Foster City, CA, USA), sICAM (Gen-Probe Diaclone SAS, Besançon, France) and ox-LDL (Mercodia Inc, Winston Salem, NC, USA).

All the patients were monitored for the development of adverse events during the study. They were evaluated at baseline and on follow-up visit using a symptom checklist. The 
study participants were provided the contact numbers of the investigators and asked to report any adverse event at the earliest. Adverse events in individual patients were evaluated via patient interviews for the presenting symptoms, physical examination and laboratory investigations. Causality assessment was carried out using the World Health Organization-Uppsala Monitoring Centre criteria and Naranjo algorithm. ${ }^{(20)}$

Data were entered in the Microsoft Office Excel 2007 database and analysed using the Statistical Package for the Social Sciences for Windows version 17 (SPSS Inc, Chicago, IL, USA). The intention-to-treat principle using data from all patients randomised for the study was followed when evaluating the efficacy and safety outcomes of treatment. Chi-square test was applied for all categorical data, and Student's $t$-test and Mann-Whitney $U$ test were used for statistical analysis of continuous data. A two-tailed p-value $<0.05$ was considered statistically significant. Data were expressed as numbers (n), percentage $(\%)$, mean \pm standard error of mean (SEM) or median (interquartile range).

\section{RESULTS}

A total of 58 patients who presented to the cardiology outpatient department were screened (after obtaining consent for screening), out of which 34 patients were found to satisfy the eligibility criteria. Of those eligible, four patients refused to participate in the study. Thus, 30 patients were enrolled in the study after written informed consent was obtained. Of these 30 patients, 10 men and 5 women (mean age $54.3 \pm 1.6$ years) received ezetimibe plus atorvastatin, while 13 men and 2 women (mean age $53.7 \pm$ 2.8 years) received only atorvastatin. One patient each dropped out from the study and control arms. The baseline characteristics of the study populatiom were comparable, and there was no statistically significant difference between the two groups with regard to variables such as age, gender or the presence of risk factors. 13 patients in the study arm and 12 in the control arm were already on low-dose atorvastatin at the time of randomisation. There was no significant difference in the baseline lipid profiles of these patients except for VLDL-C (difference 10.4 $\pm 4.5 \mathrm{mg} / \mathrm{dL}, 95 \%$ confidence interval $[\mathrm{Cl}] 1.1-19.7 ; \mathrm{p}=0.035$, Table I). Baseline hsCRP and sICAM were also not significantly different between the two groups.

In patients from the study group, TC decreased by $34.5 \%$ from $224.7 \pm 11.6 \mathrm{mg} / \mathrm{dL}$ to $147.1 \pm 11.1 \mathrm{mg} / \mathrm{dL}$ (percentage treatment difference $-14.4 \pm 6.5,95 \% \mathrm{Cl}-1.0$ to $-27.7 ; \mathrm{p}=0.041$ ), which was statistically significant when compared to the control arm in which the corresponding decrease was $20.1 \%$ from baseline (Table II). Treatment with atorvastatin plus ezetimibe also led to a considerable reduction in LDL-C, which was reduced by $44.2 \%$, from $145.3 \pm 11.4 \mathrm{mg} / \mathrm{dL}$ at baseline to $81.0 \pm 7.6 \mathrm{mg} / \mathrm{dL}$ at the end of four weeks. The reduction of LDL-C in the study group was statistically significant when compared to the control group, where LDL-C decreased by $24.3 \%$ from baseline (percentage treatment difference $-19.9 \pm 6.1,95 \% \mathrm{Cl}-7.4$ to $-32.4 ; \mathrm{p}=0.003)$.
Table I. Baseline characteristics of study participants $(n=30)$.

\begin{tabular}{lcc}
\hline Characteristic & \multicolumn{2}{c}{ No. of participants } \\
\cline { 2 - 3 } & $\begin{array}{c}\text { Atorvastatin + } \\
\text { ezetimibe }(\mathbf{n}=\mathbf{1 5})\end{array}$ & $\begin{array}{c}\text { Atorvastatin only } \\
(\mathbf{n}=\mathbf{1 5})\end{array}$ \\
\hline Age*(yrs) & $54.3 \pm 1.6$ & $53.7 \pm 2.8$ \\
Gender & & \\
Male & 10 & 13 \\
Female & 5 & 2 \\
History & & \\
Diagnosed with CAD & 11 & 12 \\
Hypercholesterolaemia & 15 & 15 \\
Diabetes mellitus & 3 & 1 \\
Hypertension & 12 & 11 \\
Tobacco use & 3 & 3 \\
Family history of CAD & 3 & 5 \\
On atorvastatin 10 mg & 13 & 12 \\
Laboratory investigation* & & \\
TC (mg/dL) & $224.7 \pm 11.6$ & $191.5 \pm 22.3$ \\
LDL-C (mg/dL) & $145.3 \pm 11.4$ & $121.6 \pm 5.4$ \\
HDL-C (mg/dL) & $44.0 \pm 1.6$ & $43.9 \pm 1.6$ \\
VLDL-C (mg/dL) & $38.8 \pm 3.4$ & $28.4 \pm 3.0^{\dagger}$ \\
TG (mg/dL) & $192.7 \pm 18.1$ & $150.1 \pm 15.3$ \\
Ox-LDL (U/L) & $76.3 \pm 8.7$ & $66.4 \pm 7.4$ \\
\hline
\end{tabular}

${ }^{*}$ Data is presented as mean \pm SEM. ${ }^{\dagger} p<0.05$.

CAD: coronary artery disease; HDL-C: high-density lipoprotein cholesterol; LDL-C: low-density lipoprotein cholesterol; ox-LDL: oxidised lowdensity lipoprotein; SEM: standard error of mean; TC: total cholesterol; TG: triglyceride; VLDL-C: very low-density lipoprotein cholesterol

In all, 13 patients on the combination treatment achieved NCEP target levels for LDL-C compared to 9 patients on atorvastatin monotherapy $(p=0.032)$.

Interestingly, patients who received atorvastatin plus ezetimibe showed an $8 \%$ reduction in HDL-C levels from baseline. However, this reduction was not significantly different from the $14.3 \%$ reduction observed in the control group (percentage treatment difference $6.3 \pm 6.6,95 \% \mathrm{Cl}-7.2$ to $19.8 ; \mathrm{p}=0.09$ ). The VLDL-C level in patients on atorvastatin plus ezetimibe changed from $38.8 \pm 3.4 \mathrm{mg} / \mathrm{dL}$ to $27.8 \pm 4.7 \mathrm{mg} / \mathrm{dL}$ at follow-up. This change was statistically significant when compared to the control group, where VLDL-C level decreased by only $3.5 \%$ after four weeks of treatment (percentage treatment difference $-24.8 \pm 10.4,95 \% \mathrm{Cl}-3.5$ to $-46.1 ; \mathrm{p}=0.016$ ). It is noteworthy that the serum TG level in patients treated with atorvastatin plus ezetimibe reduced by $25.9 \%$ from a baseline level of $192.7 \pm 18.1 \mathrm{mg} / \mathrm{dL}$. The drop in serum TG in the study arm was statistically significant when compared to the control arm, where TG increased from $150.1 \pm 15.3 \mathrm{mg} / \mathrm{dL}$ at baseline to $161.6 \pm$ $20.9 \mathrm{mg} / \mathrm{dL}$ at follow-up (percentage treatment difference $-33 \pm$ 10.3, 95\% Cl -11.9 to $-54.1 ; p=0.004)$. Ox-LDL also decreased significantly in patients receiving atorvastatin plus ezetimibe when compared to those who received only atorvastatin (percentage treatment difference $-14 \pm 6.2,95 \% \mathrm{Cl}-1.3$ to -26.7 ; $\mathrm{p}=0.030$ ) (Table II).

Serum AST, ALT, urea and creatinine were within the normal range both at baseline and at follow-up after four weeks of treatment, and there were no significant differences between the levels observed in the two groups (Table III). The median serum hsCRP level of patients in the atorvastatin plus ezetimibe 
Table II. Lipid profile of study participants at follow-up.

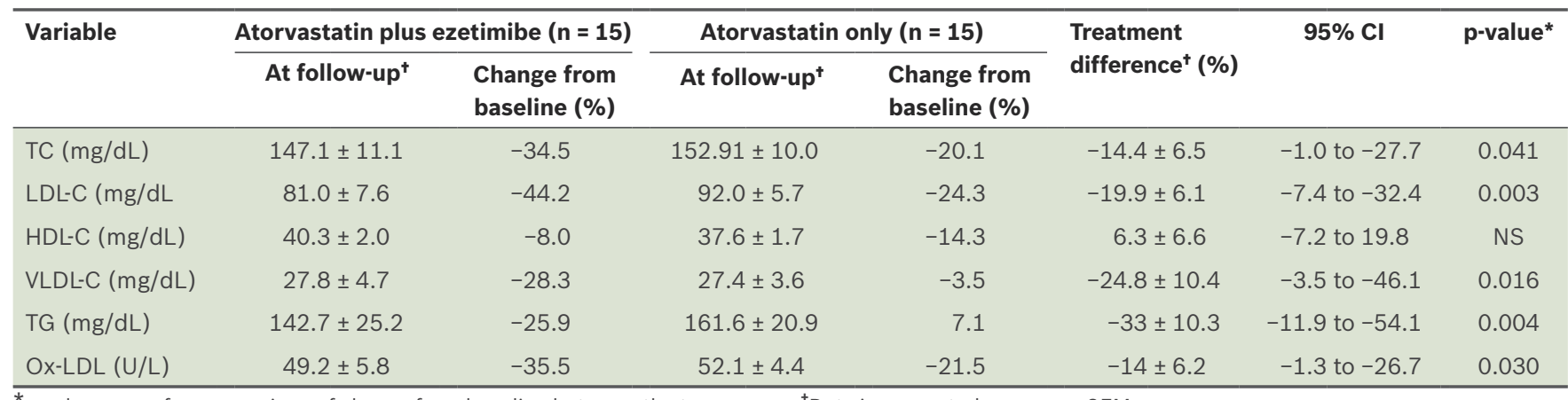

${ }^{*} \mathrm{p}$-values were for comparison of change from baseline between the two groups. ${ }^{\dagger}$ Data is presented as mean $\pm \mathrm{SEM}$

$\mathrm{Cl}$ : confidence interval; HDL-C: high-density lipoprotein cholesterol; LDL-C: low-density lipoprotein cholesterol; NS: not significant; ox-LDL: oxidised low-density lipoprotein; SEM: standard error of mean; TC: total cholesterol; TG: triglyceride; VLDL-C: very low-density lipoprotein cholesterol

Table III. Clinical biochemistry of the study participants at baseline and follow-up.

\begin{tabular}{|c|c|c|c|c|c|}
\hline \multirow[t]{2}{*}{ Variable } & \multicolumn{2}{|c|}{ Atorvastatin plus ezetimibe $(n=15)$} & \multicolumn{2}{|c|}{ Atorvastatin only ( $n=15)$} & \multirow[t]{2}{*}{ p-value* } \\
\hline & At baseline & At follow-up & At baseline & At follow-up & \\
\hline $\mathrm{AST}^{+}(\mathrm{U} / \mathrm{L})$ & $27.5 \pm 2.2$ & $28.7 \pm 1.1$ & $32.9 \pm 1.9$ & $32.2 \pm 1.3$ & NS \\
\hline $\operatorname{ALT}^{\dagger}(U / L)$ & $24.7 \pm 1.4$ & $27.1 \pm 1.6$ & $29.3 \pm 3.2$ & $27.8 \pm 2.8$ & NS \\
\hline Urea $^{\dagger}(\mathrm{mg} / \mathrm{dL})$ & $27.3 \pm 1.3$ & $26.8 \pm 1.3$ & $29.5 \pm 0.5$ & $27.5 \pm 0.8$ & NS \\
\hline Creatinine $^{\dagger}(\mathrm{mg} / \mathrm{dL})$ & $0.9 \pm 0.1$ & $0.8 \pm 0.2$ & $0.9 \pm 0.1$ & $0.9 \pm 0.1$ & NS \\
\hline $\mathrm{hsCRP}^{\text {ๆ }}$ (mg/L) & $5.3(2.0-8.3)$ & $4.9(2.7-9.4)$ & $1.4(0.8-5.3)$ & $3.0(1.2-5.7)$ & NS \\
\hline $\operatorname{sICAM}^{\pi}(\mu \mathrm{g} / \mathrm{mL})$ & $353(219-445)$ & $319(202-418)$ & $289(209-391)$ & $287(206-385)$ & 0.042 \\
\hline
\end{tabular}

${ }^{*} \mathrm{p}$-values were for comparison of change from baseline between the two groups. ${ }^{\dagger}$ Data is presented as mean \pm SEM. ${ }^{\text {"Data }}$ is presented as median (IQR). ALT: alanine transaminase; AST: aspartate transaminase; hSCRP: high-sensitivity C-reactive protein; IQR: interquartile range; NS: not significant; SEM: standard error of mean; sICAM: soluble intercellular cell adhesion molecule

group changed from $5.3 \mathrm{mg} / \mathrm{L}$ to $4.9 \mathrm{mg} / \mathrm{L}$ when compared to $1.4 \mathrm{mg} / \mathrm{L}$ and $3.0 \mathrm{mg} / \mathrm{L}$ in the control group at baseline and follow-up, respectively. However, the difference in the median serum hsCRP levels from baseline was not statistically significant between the two groups. Treatment with the combination led to a reduction in the median serum sICAM level from $353 \mu \mathrm{g} / \mathrm{mL}$ to $319 \mu \mathrm{g} / \mathrm{mL}$, and the decrease was significant when compared to patients on atorvastatin monotherapy $(p=0.042)$ (Table III).

Two patients in the study arm and one in the control arm complained of mild dyspepsia at follow-up, which was probably related to drug intake. The patients did not, however, discontinue the drug. None of the adverse events encountered in the study was serious.

\section{DISCUSSION}

The prevalence of dyslipidaemia is reported to be as high as $62 \%$ among urban Indian men, and a greater atherogenic role has been attributed to TC and LDL-C in the development of CAD. ${ }^{(3,4,21)}$ Our study was carried out to evaluate the effect of FDC of atorvastatin $10 \mathrm{mg}$ plus ezetimibe $10 \mathrm{mg}$ on the lipid profile, and ox-LDL, hsCRP and sICAM levels of Indian patients with dyslipidaemia who have CAD or are at high risk of developing CAD.

Ezetimibe plus statin inhibits both the intestinal absorption and hepatic synthesis of cholesterol, thus significantly lowering LDL-C as compared to statin monotherapy. Accordingly, treatment with a combination of atorvastatin plus ezetimibe led to a marked reduction in the levels of TC, LDL-C, VLDL-C and TG after four weeks of treatment compared to patients on atorvastatin monotherapy. Our observation regarding the reduction in TC and LDL-C levels is in agreement with other clinical trials. In the Vytorin Versus Atorvastatin (VYVA) study, for instance, the addition of ezetimibe $10 \mathrm{mg}$ to the lowest dose of a statin provided LDL-C reduction equivalent to that of three dose titrations of the statin, ${ }^{(22)}$ while in the Ezetimibe Add-on Statin for Effectiveness (EASE) trial, $10 \mathrm{mg}$ of ezetimibe added to ongoing statin therapy led to $25 \%$ reduction in LDL-C levels compared to $2 \%$ reduction with the statin alone. ${ }^{(16)}$ In addition, studies have reported that during a four-week treatment period, the degree of LDL-C reduction achieved by $40 \mathrm{mg}$ /day of atorvastatin was comparable with that obtained by $10 \mathrm{mg} /$ day of ezetimibe in combination with $10 \mathrm{mg} /$ day of atorvastatin. ${ }^{(23)}$ In our study, the proportion of patients achieving NCEP-ATP III LDL-C target levels with combination treatment was also similar to that of other reports. ${ }^{(24-27)}$

The decrease in LDL-C with combination treatment has been consistently reported across trials. Recently, the UK National Institute for Health and Clinical Excellence (NICE) assessment group carried out a meta-analysis of shorter-term studies (less than 12 weeks in duration) comparing ezetimibe coadministered with statin therapy versus statin therapy alone. It showed that the addition of ezetimibe to statin therapy reduced LDL-C concentrations by $23.2 \%$ more than statin therapy alone, and this is consistent with our observation of an additional 20\% reduction in LDL-C levels with combination treatment. ${ }^{(10)}$ Every $1 \%$ decrease in LDL-C is associated with an approximate $1 \%$ 
decrease in CAD-related mortality. ${ }^{(26)}$ Therefore, in our study, patients on combination therapy with atorvastatin plus ezetimibe presumably had an almost 20\% lower risk of developing CADrelated events than those on atorvastatin monotherapy.

Elevated ox-LDL level has also been identified as a risk factor for the development of CAD. ${ }^{(27)}$ Besides being a marker of oxidative stress, ox-LDL also increases the expression of adhesion molecules on endothelial cells, enhances monocyte chemotaxis and upregulates the genes of inflammatory mediators. ${ }^{(28,29)}$ Serum ox-LDL level decreased significantly in the patients of our study group when compared to those in the control group. However, our findings on serum ox-LDL differ from those of a recent study by Nozue et al, where significant change in ox-LDL level was not observed following the addition of ezetimibe to statin therapy. ${ }^{(30)}$ The change in ox-LDL level observed with combination therapy in our study may possibly have been because we had controlled for confounders such as diet and lifestyle patterns. ${ }^{(31)}$

No significant change in the HDL-C level was observed between the two groups in our study. This is in agreement with a study by Constance et al, where no statistically significant change in HDL-C was observed with the administration of a combination of ezetimibe $10 \mathrm{mg}$ and simvastatin $40 \mathrm{mg}$ for six weeks as compared to only atorvastatin $20 \mathrm{mg} \cdot{ }^{(32)}$ However, according to the NICE meta-analysis, the ezetimibe plus statin combination increases HDL-C levels only slightly. ${ }^{(10)}$ It is possible that a significant change in HDL-C level was not observed in our study due to its short duration and the small number of participants.

Elevated serum hsCRP is associated with a higher risk of developing CAD. The effect of statins in patients with CAD or at high risk of developing CAD is reflected by a significant reduction in hsCRP. ${ }^{(33,34)}$ In our study, the difference in the median serum hsCRP levels of patients from the study and control groups was not statistically significant. This is consistent with a recent study by Gupta et al, where ezetimibe added to stable statin therapy for 12 weeks produced no significant effect on leptin, hsCRP, tumour necrosis factor- $\alpha$ or interleukin- 6 concentrations. ${ }^{(35)}$ In contrast, Ballantyne et al, who compared atorvastatin and ezetimibe combination treatment to atorvastatin alone, reported an overall larger reduction in hsCRP levels with the combination. Interestingly, the authors found that this effect was observed by adding ezetimibe $10 \mathrm{mg}$ to a higher dose of atorvastatin $(80 \mathrm{mg})$, but not with a lower dose of atorvastatin (10 mg) despite consistent LDL-C level lowering across the whole dosing range of atorvastatin. ${ }^{(36)}$ Although currently available data suggests that ezetimibe may have a synergistic effect on hsCRP levels when combined with statins, ${ }^{(37)}$ the mechanism of this effect and the interaction of ezetimibe with different statins still require clarification. It is possible that significant change in hsCRP was not observed in our study due to its short duration.

The role of sICAM in the pathogenesis of CAD has generated considerable interest in recent years. Elevated levels of sICAM, a biomarker for inflammation, generated from endothelial cells and leukocytes have been reported in patients with stable angina and acute coronary syndrome. ${ }^{(38,39)}$ There was significant reduction in the sICAM levels of patients treated with the combination as compared to those on atorvastatin monotherapy in our study. It is possible that the reduction in SICAM with the combination therapy was associated with reductions in the level of ox-LDL, a mediator which is known to enhance the expression of cell adhesion molecules. ${ }^{(29,40)}$ Additionally, the anti-inflammatory effect could have been secondary to the LDL-C-lowering effect of the combination treatment.

Major safety concerns with statin therapy include the rare occurrence of serious muscle-related adverse events and the potential of elevated serum transaminases. A study on 4,558 patients receiving the ezetimibe-simvastatin combination concluded that the incidence of myopathy $(0.04 \%)$ was no more common in these patients than that observed in 2,563 patients taking simvastatin alone (0.08\%). In addition, there was no report of rhabdomyolysis in either group. ${ }^{(41)}$ Furthermore, according to Florentin et al, neither ezetimibe monotherapy nor combination treatment has been related to liver failure, the need for liver transplantation or death. ${ }^{(42)}$

The FDC of atorvastatin plus ezetimibe in our study was well-tolerated, with a safety profile comparable to that of atorvastatin monotherapy. Mild dyspepsia was the only adverse event encountered in this study. There were no clinically significant alterations in AST, ALT, urea and creatinine levels in both patient groups. Our observations were in accordance with other studies that reported similar safety profiles for the ezetimibe-statin combination and statin monotherapy, even in special populations such as elderly patients and those with chronic kidney disease. ${ }^{(22,43,44)}$

In conclusion, our study suggests that atorvastatin plus ezetimibe combination therapy has relatively better lipid-lowering and anti-inflammatory effects than atorvastatin monotherapy, with a comparable safety profile. To our understanding, this is likely the first randomised, double-blind, comparative trial of the combination in Indian patients with dyslipidaemia who have CAD or are at risk of developing CAD. The reduction of LDL-C with atorvastatin plus ezetimibe combination therapy in Indian patients, as observed in our study, was comparable to that observed in non-Indian patients of other races and ethnicities. ${ }^{(45)}$ However, the limitations of our study, including a small sample size, short duration, underrepresentation of women and patients with diabetes mellitus in the study groups, and the fact that most of the patients were already on low-dose atorvastatin at study initiation, should be noted. Nevertheless, this pilot study may be useful in providing a background for further studies aiming at a comprehensive evaluation of the combination treatment in Indian patients.

\section{ACKNOWLEDGEMENTS}

This study was supported by Torrent Research Centre, Ahmedabad, India. We thank Dr Abmrish Srivastava of Torrent Pharmaceuticals Ltd, Ahmedabad, for his assistance in conducting 
this study. We are also thankful to Dr Surender Singh, Mr Vinod Nair and Mr Rohit of the Department of Pharmacology, AlIMS, New Delhi, for their help during analysis of the samples.

\section{REFERENCES}

1. Sharma M, Ganguly NK. Premature coronary artery disease in Indians and its associated risk factors. Vasc Health Risk Manag 2005; 1:217-25.

2. Goyal A, Yusuf S. The burden of cardiovascular disease in the Indian subcontinent. Indian J Med Res 2006; 124:235-44.

3. Gupta R, Gupta VP, Sarna M, et al. Prevalence of coronary heart disease and risk factors in an urban Indian population: Jaipur Heart Watch-2. Indian Heart J 2002; 54:59-66.

4. Srinath Reddy K, Shah B, Varghese C, Ramadoss A. Responding to the threat of chronic diseases in India. Lancet 2005; 366:1744-9.

5. Nixon JV. Who should receive a statin drug to lower cardiovascular risk? Does the drug and the dose of the drug matter? Vasc Health Risk Manag $2006 ; 2: 441-6$

6. Rutishauser J. The role of statins in clinical medicine-LDL-cholesterol lowering and beyond. Swiss Med Wkly 2006; 136:41-9.

7. Davignon J, Leiter LA. Ongoing clinical trials of the pleiotropic effects of statins. Vasc Health Risk Manag 2005; 1:29-40.

8. Liao JK. Isoprenoids as mediators of the biological effects of statins. J Clin Invest 2002; 110:285-8.

9. Miida T, Hirayama S, Nakamura Y. Cholesterol-independent effects of statins and new therapeutic targets: ischemic stroke and dementia. J Atheroscler Thromb 2004; 11:253-64.

10. Hamilton-Craig I, Kostner K, Colquhoun D, Woodhouse S. Combination therapy of statin and ezetimibe for the treatment of familial hypercholesterolemia. Vasc Health Risk Manag 2010; 6:1023-37.

11. Grundy SM, Cleeman JI, Merz CN, et al. Implications of recent clinical trials for the National Cholesterol Education Program Adult Treatment Panel III Guidelines. J Am Coll Cardiol 2004; 44:720-32.

12. Backes JM, Gibson CA, Howard PA. Optimal lipid modification: the rationale for combination therapy. Vasc Health Risk Manag 2005; 1:317-31.

13. Neal RC, Jones PH. Complementary therapy to target LDL cholesterol: the role of the ezetimibe/simvastatin combination. Vasc Health Risk Manag 2006; 2:31-8.

14. Al Badarin FJ, Kullo IJ, Kopecky SL, Thomas RJ. Impact of ezetimibe on atherosclerosis: is the jury still out? Mayo Clin Proc 2009; 84:353-61.

15. Kastelein JJ, Akdim F, Stroes ES, et al. Simvastatin with or without ezetimibe in familial hypercholesterolemia. N Engl J Med 2008; 358:1431-43.

16. Pearson TA, Denke MA, McBride PE, et al. A community-based, randomized trial of ezetimibe added to statin therapy to attain NCEP ATP III goals for LDL cholesterol in hypercholesterolemic patients: the ezetimibe add-on to statin for effectiveness (EASE) trial. Mayo Clin Proc 2005; 80:587-95.

17. Landmesser $U$, Bahlmann F, Mueller M, et al. Simvastatin versus ezetimibe: pleiotropic and lipid-lowering effects on endothelial function in humans. Circulation 2005; 111:2356-63.

18. Jayaram S, Jain SD, Bhatia G, et al. Assessment of the efficacy, safety and tolerability of ezetimibe $10 \mathrm{mg}$ in adult patients with primary hypercholesterolemia: The first Indian Study. Indian Pract 2004; 57:73-9.

19. Athyros VG, Tziomalos K, Kakafika Al, et al. Effectiveness of ezetimibe alone or in combination with twice a week atorvastatin $(10 \mathrm{mg})$ for statin intolerant high-risk patients. Am J Cardiol 2008; 101:483-5.

20. Farcas A, Bojita M. Adverse drug reactions in clinical practice: a causality assessment of a case of drug-induced pancreatitis. J Gastrointestin Liver Dis 2009; 18:353-8.

21. Mohan V, Deepa R, Rani SS, Premalatha G, Chennai Urban Population Study (CUPS No.5). Prevalence of coronary artery disease and its relationship to lipids in a selected population in South India: The Chennai Urban Population Study (CUPS No. 5). J Am Coll Cardiol 2001; 38:682-7.

22. Ballantyne CM, Abate N, Yuan Z, King TR, Palmisano J. Dose-comparison study of the combination of ezetimibe and simvastatin (Vytorin) versus atorvastatin in patients with hypercholesterolemia: the Vytorin Versus Atorvastatin (VYVA) study. Am Heart J 2005; 149:464-73.

23. Piorkowski M, Fischer S, Stellbaum C, et al. Treatment with ezetimibe plus low-dose atorvastatin compared with higher-dose atorvastatin alone: is sufficient cholesterol-lowering enough to inhibit platelets? J Am Coll Cardiol 2007; 49:1035-42.
24. Conard S, Bays H, Leiter LA, et al. Ezetimibe added to atorvastatin compared with doubling the atorvastatin dose in patients at high risk for coronary heart disease with diabetes mellitus, metabolic syndrome or neither. Diabetes Obes Metab 2010; 12:210-8.

25. Zieve F, Wenger NK, Ben-Yehuda O, et al. Safety and efficacy of ezetimibe added to atorvastatin versus up titration of atorvastatin to $40 \mathrm{mg}$ in Patients $>$ or $=65$ years of age (from the ZETia in the ELDerly [ZETELD] study). Am J Cardiol 2010; 105:656-63.

26. Spratt KA. Reducing the risk of coronary heart disease via lipid reduction. J Am Osteopath Assoc 2004; 104:S9-13.

27. Grigore L, Norata GD, Catapano AL. Combination therapy in cholesterol reduction: focus on ezetimibe and statins. Vasc Health Risk Manag 2008; 4:267-78.

28. Mitra S, Deshmukh A, Sachdeva R, Lu J, Mehta JL. Oxidized low-density lipoprotein and atherosclerosis implications in antioxidant therapy. Am J Med Sci 2011; 342:135-42.

29. Nagy L, Tontonoz P, Alvarez JG, Chen H, Evans RM. Oxidized LDL regulates macrophage gene expression through ligand activation of PPARgamma. Cell 1998; 93:229-40.

30. Nozue T, Michishita I, Mizuguchi I. Effects of ezetimibe on remnant-like particle cholesterol, lipoprotein (a), and oxidized low-density lipoprotein in patients with dyslipidemia. J Atheroscler Thromb 2010; 17:37-44.

31. Fonseca HA, Izar MC, Bianco HT, Fonseca FA. Ezetimibe, oxidized low density lipoprotein, Lp (a), and dyslipidemia. J Atheroscler Thromb 2010; 17:888

32. Constance C, Westphal S, Chung N, et al. Efficacy of ezetimibe/simvastatin $10 / 20$ and $10 / 40 \mathrm{mg}$ compared with atorvastatin $20 \mathrm{mg}$ in patients with type 2 diabetes mellitus. Diabetes Obes Metab 2007; 9:575-84.

33. Ridker PM, Cannon CP, Morrow D, et al. C-reactive protein levels and outcomes after statin therapy. N Engl J Med 2005; 352:20-8.

34. Balk EM, Lau J, Goudas LC, et al. Effects of statins on nonlipid serum markers associated with cardiovascular disease: a systematic review. Ann Intern Med 2003; 139:670-82.

35. Gupta M, Szmitko PE, Tsigoulis M, et al. Effects of ezetimibe add-on to statin therapy on adipokine production in patients with metabolic syndrome and stable vascular disease. J Cardiovasc Pharmacol 2010; 56:241-5.

36. Ballantyne CM, Houri J, Notarbartolo A, et al. Effect of ezetimibe coadministered with atorvastatin in 628 patients with primary hypercholesterolemia: a prospective, randomized, double-blind trial. Circulation 2003; 107:2409-15.

37. Kinlay S. Low-density lipoprotein-dependent and -independent effects of cholesterol-lowering therapies on C-reactive protein: a meta-analysis. J Am Coll Cardiol 2007; 49:2003-9.

38. Blankenberg S, Rupprecht HJ, Bickel C, et al. Circulating cell adhesion molecules and death in patients with coronary artery disease. Circulation. 2001; 104:1336-42.

39. Wijnstok NJ, Twisk JW, Young IS, et al. Inflammation markers are associated with cardiovascular diseases risk in adolescents: the Young Hearts project 2000. J Adolesc Health 2010; 47:346-51.

40. Rosenson RS, Wolff D, Tangney CC. Statins reduce oxidized low-density lipoprotein levels, but do not alter soluble intercellular cell-adhesion molecule-1 and vascular cell-adhesion molecule- 1 levels in subjects with hypercholesterolaemia. Clin Sci (Lond) 2004; 106:215-7.

41. Davidson MH, Maccubbin D, Stepanavage M, Strony J, Musliner T. Striated muscle safety of ezetimibe/simvastatin (Vytorin). Am J Cardiol 2006; 97:223-8.

42. Florentin M, Liberopoulos EN, Elisaf MS. Ezetimibe-associated adverse effects: what the clinician needs to know. Int J Clin Pract 2008; 62:88-96.

43. Foody JM, Brown WV, Zieve F, et al. Safety and efficacy of ezetimibe/ simvastatin combination versus atorvastatin alone in adults $\geq 65$ years of age with hypercholesterolemia and with or at moderately high/high risk for coronary heart disease (the VYTELD study). Am J Cardiol 2010 106:1255-63.

44. Denke M, Pearson T, McBride $P$, et al. Ezetimibe added to ongoing statin therapy improves LDL-C goal attainment and lipid profile in patients with diabetes or metabolic syndrome. Diab Vasc Dis Res 2006; 3:93-102.

45. Pearson TA, Denke MA, McBride PE, et al. Effectiveness of ezetimibe added to ongoing statin therapy in modifying lipid profiles and low-density lipoprotein cholesterol goal attainment in patients of different races and ethnicities: a substudy of the Ezetimibe add-on to statin for effectiveness trial. Mayo Clin Proc 2006; 81:1177-85 